\title{
CLOSE ENCOUNTERS:
}

\section{CREATING A SAFE SPACE FOR \\ INTERCULTURAL BIBLE READING ${ }^{1}$ \\ PART II}

\author{
Charlene van der Walt \\ Department of Old and New Testament \\ Stellenbosch University
}

\begin{abstract}
Theoretically the process of intercultural Bible reading should create a safe space where the voice of the individual can be heard in community with others. It should be a space where the individual is not only free to speak but also to have the innate experience of truly being heard. In this respect the intercultural Bible reading experience becomes a space that promotes human dignity and has the inherent capacity to facilitate social transformation. Although these Bible study groups can ideally be a safe space with the potential for social transformation, the practical reality shows a more complicated dynamic. Two important factors that contribute to the complexity are the ideological framework of individual participants and the underlying power dynamic in the social interaction. To bring the concepts of power and ideology in intercultural Bible reading into focus, an empirical study was conducted. Building on the theoretical discussion in Part I, this contribution will turn to the empirical study. An overview will be provided of the carefully constructed research process which allowed participants time for personal reflection, intimate dialogue as well as group discussion in order to create a free and safe space for creative interaction. In order to critically engage with the data collected, a unique set of qualitative data analysis tools was developed to deal with the issues of power and ideology as mentioned above. The final part of the article will discuss this process of qualitative data analysis and give a brief summary of the research results.
\end{abstract}

Key Words: Intercultural Bible Reading, Power, Ideology

\section{A Space Created}

In order to engage with the intercultural Bible reading space in a comprehensive manner, the choice was made for a qualitative approach. ${ }^{2}$ The main criteria for the research design were:

\footnotetext{
Edited version of a paper delivered at the Seventh International Congress of Qualitative Inquiry, held at the University of Illinois at Urbana-Champaign, Illinois, USA, 18-21 May 2011.

2 Fundamentally a qualitative research design was chosen because of the possibilities it held with regards to the research questions. Both the research questions were interested in an in-depth analysis of phenomena in particular social circumstances. The following underlining principles of qualitative research made it clear that this type of research design was what was required to engage accurately with the research questions. 1) Truth is relative. "Qualitative researchers generally subscribe to the adage that 'if people believe things to be real,
} 
- Interculturality: The key moment that the research wanted to engage, was intercultural in nature. Firstly, the research groups had to contain members from different cultural, socio-economic and educational backgrounds. The diversity of the groups had to be optimal in order to ensure that participants were truly met by the 'other'. A second intercultural moment that the research wanted to facilitate was that of participants' meeting the culturally removed Biblical text. Participants needed to be lead through a process in which they discovered that the biblical story was one that originated in a foreign cultural context. Sufficient time needed to be given for both of these moments to take place.

- Relationship to Biblical Text: Participants join the intercultural Bible reading space from a variety of positions. Some participants are devout believers and the Bible plays a central role in their religious practice. This is, however, not the case for all participants. The research design needed to create a space for participants from different positions.

- Observing the power dynamic: In accordance with Foucault's theory on power the strategies in a social environment needed to be observed. The research design had to allow enough time for interaction between the participants to be observed.

- Shift in ideology: Because the research question was focused on change in the case of the ideological position of participants a process needed to be developed that tested the starting ideological framework of participants, that allowed for in-depth intercultural exposure and finally tested the ideological position of participants again at the end of the engagement.

- The Possibility for Change: In order to harbour an environment for inclusive thinking ${ }^{3}$ where change could be possible, the research design needed to be structured in such a way that participants felt safe, protected and cared for in the space that was created. The encounter with the 'other' can often be threatening and participants may harbour fear with regards to this encounter. Participants needed to be reminded of a shared humanity underneath the surface of difference.

The physical research design was developed, bearing these core considerations in mind.

then they are real in their consequences" (Barbour 2008:28). The question is therefore rather about the perception that a research subject has in a particular set of circumstances and how that perception influences behaviour. 2) Qualitative research methods allow for multiple realities. "The role of qualitative research is not to determine which account is the more accurate or 'truthful', but is rather to use these accounts as a resource in order to understand how 'situated accounts' are told in a way that allow speakers to achieve a different purpose through emphasizing some aspects of their stories and de-emphasizing others" (Barbour 2008:28). 3) Research positions are not static, but due to the fluidity of the research process it is subject to change. Qualitative methods, by virtue of their immediacy and capacity to encourage respondents to question their ideas, may elicit contradictory remarks - even in the space of one interview or focus group discussion" (Barbour 2008:29). 4) Qualitative research methods focus on words not on numbers. "Qualitative research places emphasis on understanding through looking closely at people's words, actions and records. The traditional or quantitative approach to research looks past these words, actions and records to their mathematical significance" (Maykut \& Morehouse 1994:27). 5) Qualitative research aims to discover and describe. "The goal of qualitative research is to discover patterns which emerge after close observation, careful documentation, and thoughtful analysis of the research topic. What can be discovered by qualitative research is not sweeping generalizations, but contextual findings. This process of discovery forms the basis of the philosophic underpinning of the qualitative approach" (Maykut \& Morehouse 1994:21).

$3 \quad$ Kool (2004:370) remarks in this regard: "In the extension of the capacity to change perspective lies the capacity for inclusive thinking. Inclusive thinking means that one experiences and approaches another as someone with the same human dreams and longings for a safe, happy and full life." 


\section{Research Population}

As mentioned, the research took place in Grahamstown, a culturally diverse semi-rural area, in the Eastern Cape province of South-Africa. The reason for conducting the research in Grahamstown was that my position in the community, as the Dutch Reformed minister of religion from 2006 to May 2010, facilitated my access to research subjects. My regular contact with religious leaders enabled me to identify research subjects that represented diversity in terms of race, age, level of education, cultural background, sexual orientation, socio-economic position, relational status and faith tradition. Each research group consisted of eight members, thus allowing for optimal diversity but not compromising intimacy and personal space. The research process was conducted with four different groups. Participants were chosen to more or less represent the following profile. ${ }^{4}$

\begin{tabular}{|l|l|}
\hline Profile number & Description of profile \\
\hline Profile 1 & $\begin{array}{l}\text { Young participant in secondary education, preferably between the age } \\
\text { of 16-18 }\end{array}$ \\
\hline Profile 2 & $\begin{array}{l}\text { A skilled reader within formal ministry of with some level of } \\
\text { theological education. }\end{array}$ \\
\hline Profile 3 & An older participant from the Dutch reformed tradition. \\
\hline Profile 4 & A participant from the Anglican faith community. \\
\hline Profile 5 & A black, Xhosa speaking woman. \\
\hline Profile 6 & A non-believer. \\
\hline Profile 7 & A younger woman (28-40 years) from the Afrikaans community. \\
\hline Profile 8 & $\begin{array}{l}\text { A wild card that consisted of anyone that would further enrich diversity } \\
\text { in the group. }\end{array}$ \\
\hline
\end{tabular}

\section{Research Environment}

The research discussions were conducted in the rectory of the Dutch Reformed church in Grahamstown. The spacious environment was ideally suited to facilitate the group discussion as well as the interactive sessions. The grounds of the Dutch Reformed church also allowed for space for quiet reflection and contemplation. The area where the research was conducted was set up in such a way as to allow for optimal interaction and data collection.

\section{Qualitative Research Assistant}

I had the privilege to work with a very skilled research assistant on the project, who was situated in a semi-participatory position in the research process. The research assistant guided the data collection process whilst I had the opportunity to be a non-participating observer.

\footnotetext{
4 The profile spectrum as set out in the table above functions only as a broad guideline for the composition of the research groups. A certain amount of flexibility was allowed in order to get the balance of the groups correct.

5 By including a skilled reader to each group the importance of the interaction between skilled and lay readers in the intercultural Bible reading process is reiterated.
} 


\section{Research Process}

The process that was conducted with each group in order to allow for maximum exposure to the culturally diverse other and the culturally removed biblical text, was structured in the following way:

Phase 1: Introduction and meeting of fellow participants: Each participant was met by the research team and introduced to fellow participants. Participants received a workbook ${ }^{6}$ that contained all the information for data collection and a nametag that would allow for easy identification of participants. After allowing for some time to socialise and enjoy a quick cup of tea, participants were encouraged to fill in the demographic questionnaire on the first pages of the workbook. When the entire group was present, the research procedure was explained to the group and all questions were answered pertaining to the research set-up. Participants were ensured of the protection of their privacy and encouraged to share openly in the discussions as well as in the workbook.

Phase 2: Participants were afforded the opportunity to complete a demographic questionnaire. After completion participants were guided through an exercise to determine their initial ideological position. Images, chosen in accordance with Hofstede's cultural theory, ${ }^{7}$ were flashed on a screen as impulses to encourage participants to free write for one minute. Participants were encouraged to write without censoring their response, but rather to just give their first gut level response to the images. This exercise was done twice in the research process. It was done once at the beginning to acquire a basic understanding of the participants' ideological position and once at the end of the process to determine whether any change could be noted after completion of the research.

Phase 3: Timeline exercise - in order to indicate the simultaneous diversity and connectedness of the group members, an exercise was conducted that enabled participants to plot themselves temporarily and contextually. A paper timeline was fixed against one of the rectory walls that showed images of important dates. Participants were then given three cards on which they had to indicate: 1) the year in which they were born, 2) where they were in $1994^{8}$ and 3) when it was that they became aware for the first time of violence against women as an issue. Participants were then given an opportunity to add their cards to the timeline and explain their experiences to the rest of the group.

Phase 4: Lectio divina - The biblical text of 2 Samuel 13:1-22 that would serve as the basis for the intercultural conversation, was introduced to participants by a slow, repeated, contemplative reading. The Lectio divina process enables participants to

6 In order to streamline the data-collection process a workbook was constructed that contained: a letter explaining the research focuses and procedure, all the questionnaires, various translations of 2 Samuel 13:1-22 as the base text for the intercultural discussion, space to make notes in the research process and all other relevant research material. The workbook was printed in A4 format to allow easy readability and enough space for notes and reflection. The workbook was beautifully illustrated by artist Tammy Griffin.

7 Hofstede's cultural theory, as discussed above, identifies five depth dimensions of culture. Hofstede's theory represents culture as linear axes indicating the continuum between two opposite poles in each depth dimension. Two images per depth dimension were chosen to test participants' basic ideological response (i.e. words located in a specific social context) before and after the intercultural engagement.

81994 was a watershed year in the history of South Africa indicating the end of the apartheid regime as the first general elections were held. Fundamentally it was this change in the country that allowed for the possibility of intercultural engagement. 
gradually listen to the story and to hear different aspects in the repeated reading. Participants were encouraged to make cryptic notes of their first reactions to the text.

Phase 5: Biblical Timeline - To place Tamar's story in a cultural and temporal framework a brief description of the so-called 'world behind the text ${ }^{\text {'9 }}$ was compiled and placed in the workbook for participants to read in their own time.

Phase 6: Personal reflection - In order to allow participants some time to read the background information of the text and some time for personal reflection, a quiet tea/coffee break was afforded the group. Participants were not allowed conversation with each other during this time, but rather asked to respect each other and allow for some time for personal reflection.

Phase 7: One-on-one listening experience - Before the official group discussion took place, individual participants were paired off with a culturally diverse other for a one-on-one conversation. Each participant was granted a couple of minutes to share their reaction on the story with their partner. No discussion was allowed at this point, participants were encouraged rather to listen to the other and maybe make notes on what they heard for further discussion. The aim of the exercise was to expose participants to the interpretation of a culturally diverse other, but to minimize any opportunity for judgment.

Phase 8: Group discussion - participants were now allowed time to share their opinions on the text and related issues in a free and open space created by the research assistant. All group discussions were started with a very simple question namely: "What was it in the story that we read together that touched you the most?" Participants were then encouraged to share their views allowing for everyone to take part as they personally felt comfortable. An audio recording that was made of the conversations was later transcribed and it formed the basis for the conversation analysis to determine the power dynamic that functioned in the group discussions.

Phase 9: After the group discussion came to a close, participants filled in a final questionnaire that pertained to the power dynamics that participants noticed in the story of Tamar as well as to their experience of the power dynamic in the group discussion. Participants were then lead through the same exercise as in the beginning where the images chosen according to Hofstede's theory on culture were flashed on the screen. Participants were asked to free write for a period of time, taking into account the influence (if any) of the time spent in the research process.

Phase 10 The process was drawn to a close, allowing participants time for questions and contributions. They were thanked for all their time and effort.

9 These notes describe the cultural world and the ideological motivations in which the writers of the text functioned. 
The time scheduling for the research process was structured as follows:

\begin{tabular}{|l|l|}
\hline Activity & Time allowed \\
\hline Introduction, completion of demographic questionnaire & $10 \mathrm{~min}$. \\
\hline Ideological testing exercise & $15 \mathrm{~min}$. \\
\hline Timeline exercise & $30 \mathrm{~min}$. \\
\hline Lectio divina & $20 \mathrm{~min}$. \\
\hline Personal reflection & $20 \mathrm{~min}$. \\
\hline 1on1 & $15 \mathrm{~min}$. \\
\hline Group discussion & $40 \mathrm{~min}$. \\
\hline Completing questionnaire and second ideological testing exercise & $30 \mathrm{~min}$. \\
\hline
\end{tabular}

The physical research was conducted on 23 and 30 May 2009. To accommodate the four different groups that participated in the research, two daily sessions were conducted. A morning session from 9:00 to 12:00 and an afternoon session from 14:00 to 17:00 were held. The groups consisted of eight participants chosen according to the research profiles described above. All the participants arrived on time and the research process ran according to schedule in each of the sessions. After the process the participants from each group handed in their completed workbooks, containing the raw data of the study.

All the data was converted into a workable digital format and the four conversations were transcribed. The data in digital format was then analyzed and interpreted.

\section{A Space Analyzed}

In order to analyze the raw data that was collected during the empirical data collection sessions, unique data analysis tools needed to be developed.

\section{Ideological analysis}

As described above, the ideological positions of participants were tested, using images chosen according to Hofstede's depth dimensions of culture. In order to illustrate the development of the data analysis tool, I will use one of the images as an example.

The first depth dimension that Hofstede identifies is power distance: "Power distance can therefore be defined as the extent to which the less powerful member of institutions and organizations within a country expect and accept that power is distributed unequally" (Hofstede 1991:28). The classic superior/subordinate relationships present in all societies were represented by this dimension. One of the images chosen to test individual participants' position in terms of power dynamics in a society was: 


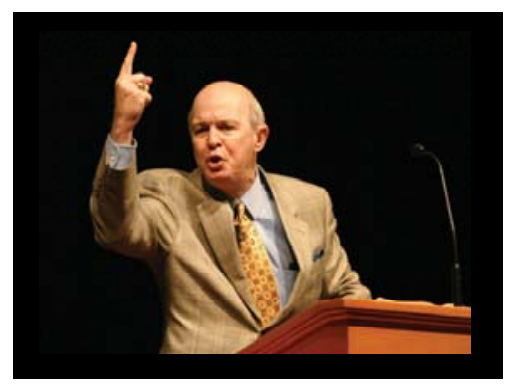

Image $1^{10}$

The image was chosen because of the strong patriarcal leadership style that it portrayed. The image shows something of the traditional top-down style of leadership that is outocratic and does not allow for negotiation or conversation.

The continuum that Hofstede suggests for this depth dimension is:

Hofstede (1991:27) describes the difference between the extreme poles as follows: "In small power distance countries there is limited dependence of subordinates on bosses, and a preference for consultation, that is interdependence between boss and subordinate. The emotional distance between them is relatively small: subordinates will quite readily approach and contradict their bosses. In large power distance countries there is considerable dependence of subordinates on bosses. Subordinates respond by either preferring such dependence or rejecting it entirely, which in psychology is known as counterdependence: that is dependence, but with a negative sign. Large power distance countries thus show a pattern of polarization between dependence and counterdependence. In these cases, the emotional distance between subordinates and their bosses is large: subordinates are unlikely to approach and contradict their bosses directly." In terms of Image 1 the difference can be illustrated as follows:

Participant identifies figure as one with power./Participant rejects style of leadership completely.
Participants do not identify figure as a person with power./Power in leadership is situated in a different style.

In the free writing exercise that was conducted in the research process, participants had the opportunity to respond to the images before the start of the intercultural engagement and after the process had been completed. Using these gut level responses, participants who provided their positions were plotted on graphic axes. The position of the participants before the start of the process was indicated with an (A) and the position after the process was completed with a (B).

\section{Depth dimensions of culture according to Hofstede}

Extreme pole A

Extreme pole B

10 All images used in the study were obtained from www.google-image.com and were not in any way protected by copyright. 
Participants' individual positions were then indicated on a diagram containing all the graphic axes related to the images chosen according to Hofstede's depth dimensions of culture. Responses were plotted in the following ways:

A. In the case where no substantial shift had occurred in the response of participants before and after the process, the $\mathrm{A}$ and $\mathrm{B}$ responses were indicated at the same place:

Depth dimensions of culture according to Hofstede

AB

Extreme pole A

Extreme pole B

B. In the case of an extreme shift in position before and after the process:

Depth dimension of culture according to Hofstede

A

Extreme pole A
B

Extreme pole B

C. The most frequent pattern observed in the analyzing of the data was not an extreme shift from $\mathrm{A}$ to $\mathrm{B}$, but rather that the $\mathrm{B}$ response showed a greater awareness related to issues that were discussed in the group conversations. Graphically this was indicated in the following way:

Depth dimension of culture according to Hofstede

AB

Extreme pole A

Extreme pole B

The diagram containing all the A and B responses of each individual participant showed whether shifts took place in participants during the process in an easy understandable way. The diagrams functioned as the core data for the research results on the ideological position of individual participants.

\section{Power Dynamics Analysis}

The power dynamics which functioned in the intercultural conversations that the groups had amongst themselves were analyzed with the help of the qualitative data analysis tool of conversation analysis. "Conversation analysis is an approach to the study of talk in interaction which grew out of the ethnomethodological tradition in sociology developed by Harold Garfinkle $(1964,1967,1988)$. Ethnomethodology as a field of sociology studies the common sense resources, practices and procedures through which members of a society produce and recognize mutually intelligible objects, events and courses of action" (Liddicoat 2007:2). Liddicoat opines that the focus of Garfinkle's work was: “...to study the social structure of everyday lived experience and to develop an understanding of how structures of everyday activities are ordinarily and routinely produced and maintained." The focus of conversation analysis closely matches Foucault's theory on power, namely that it is not a commodity that someone/something possesses but rather a strategy that functions in a social dynamic. 
Using the principles of conversation analysis, the patterns that existed in the conversations were identified and described. ${ }^{11}$

- Firstly, the transcribed conversations were subjected to a turn-taking analysis paying attention to the contributions of each individual participant. Each utterance by a participant was then coded as follows:

\begin{tabular}{|l|l|}
\hline Textual tool & Code \\
\hline Text & Emotional response \\
\hline Text & Personal experience/story \\
\hline Text & Remark related to the biblical text \\
\hline Text & Explicit reference to other participant \\
\hline
\end{tabular}

The theme of each utterance was identified and it was indicated how the present utterance of an individual related to that which had preceded it.

- Secondly, a mechanical analysis was done of each conversation indicating the number of times that an individual participated in the conversation and the number of words used by a participant. These results were then connected to the ideological shifts/lack of shifts that an individual showed as a result of the ideological analysis.

- Finally, a theme analysis was done of the conversation, showing the main themes in the conversation and also indicating which individuals participated in the conversational development of the theme.

\section{A Space Evaluated}

After conducting the empirical research and analyzing the data obtained from the qualitative research design, the following conclusions were made regarding the intercultural Bible reading space:

- Using the data analysis tool that was designed by using Hofstede's depth dimensions of culture, a broad cultural profile could be constructed of the women who participated in the study. This broad cultural definition became the background against which individual participants' ideological position could be interpreted. The broad cultural position of women who took part in the study can be described as follows: Respondents who took part in this study are embedded in a large power distance cultural context. Religion and technology serve as tools to minimize the fear and anxiety that uncertainty implies. The dominant collective framework in which the woman functions does not negate individual aspirations for personal success. The women function in a masculine

11 Ten Have (1999:110-119) describes three possible strategies that could be used to identify and analyze the patterns that manifest in a conversation: "a) Turn taking organization: The idea of turn taking as an organized activity is one of the core ideas of conversation analysis. As Sacks has observed, the basic fact about conversation is that, overwhelmingly, there is one and only one person speaking at a time, while speaker change recurs with minimal gap and minimal overlap." Speakers respond one after the other and the transitions is almost organic. There are three ways in which the transitions usually take place between speakers: "(A) next speaker can be selected by the previous one, speakers can self select, or the present speaker can continue speaking. b) Sequence organization: Utterances in interactional talk are sequentially organized. The idea of 'sequence' refers to the common experience that one thing can lead to another. For conversation, this means that any utterance in interaction is considered to have been produced for the place in the progression of the talk where it occurs, especially just after the preceding one, while at the same time it creates a context for its own next utterance. c) Repair organization: organized ways of dealing with various kinds of trouble in the interaction's progress, such as problems of (miss)hearing or understanding." 
culture where the role differentiation between the sexes is very rigid. Homework and childrearing are tasks that are traditionally associated with the female gender role. Both traditional African and Western values function in the society where the women function.

The diagram that contained a graphic representation of the individual's response to the images chosen according to Hofstede's depth dimensions of culture indicated the ideological shifts/lack of shifts that participants underwent in the course of the intercultural process. The result of the ideological analysis showed that a substantial number of participants showed a shift in position after the intercultural engagement. Although the shifts that participants made were not radical in nature, there was a remarkable increase in awareness pertaining to issues that were discussed in the intercultural conversations. The conclusion was drawn that those individuals that engage with the culturally diverse other and the culturally removed biblical text show a greater awareness of the complexities involved in symbolic forms. Intercultural conversation can therefore be successfully used as a tool to create an awareness of social problematic within individuals. The encounter of the 'other' and the social realities of the 'other' create a greater awareness of the personal position that individuals have in a certain society, especially if the position is one of privilege. Intercultural conversation is thus a meaningful tool to create a greater sense of social awareness.

- The second question that the research engaged with was related to the power dynamic that could be observed in the intercultural Bible reading space. The theory on power by Foucault and conversation analysis were used as a tool to conduct the research; each conversation was analyzed. Each conversation had its own unique character due to the personalities of the individuals who participated in the conversation. Even though the criteria for each group were consistent, the influence of personality, communication style and religious framework made for completely different group dynamics. The dynamic of the group was thus closely related to the individuals who were present in the group. In each group there were dominant individuals, alliances and discourses that developed due to the composition of the group and can only be explained against that background.

The conclusion of the conversation analysis can be summed up as follows: the intercultural bible reading conversation cannot escape the realities of an underlying power dynamic. Power functions in social contexts and cannot be wished away or ignored. Consideration must be given to this reality in the composition of groups. Cultural diversity should not be the only criteria for the composition of groups, but a range of other factors should be taken into account. ${ }^{12}$

The intercultural Bible reading space, theoretically based on the combined frameworks of Feminism and African hermeneutic, is a meaningful communal space that allows for the unique voice of the individual to be heard. It is furthermore a space that challenges individual ideologically and thus creates a greater awareness of the complex nature of symbolic forms within a specific social context. The social awareness of individuals

12 Alliances easily develop between individuals that share similar life experiences, background or knowledge. The forming of alliances cannot be avoided and therefore it is important also to notice the communalities that participants share when compiling the research groups. Alliances that develop can easily become dominant and exclusive, thus sidelining more diverse participants. When compiling a group, a fine balance is needed. 
broadens through the engagement with the culturally diverse 'other' and the culturally removed biblical text. It is, however, also important to realize that the intercultural Bible reading space is complex and cannot avoid the functioning of a power dynamic.

\section{BIBLIOGRAPHY}

Barbour, RS 2008. Introducing qualitative research: A student's guide to the craft of doing qualitative research. London: Sage Publications.

Hofstede, GH 1991. Cultures and organizations: Software of the mind. London: McGraw-Hill.

Kool, M 2004. Intercultural Bible reading as a practical setting for intercultural communication, in De Wit, H, Jonker, LC, Kool, M \& Schipani, D (eds.). Through the eyes of another: Intercultural reading of the Bible. Indiana: Institute of Mennonite Studies. 360-376.

Liddicoat, AJ 2007. An introduction to conversation analysis. London: Continuum.

Maykut, P \& Morehouse, R 1994. Beginning qualitative research: A philosophic and practical guide. London: The Falmer Press.

Ten Have, P 1999. Doing conversation analysis: A practical guide. London: Sage Publication. 